\title{
Diversidade, estrutura e distribuição espacial de palmeiras em um cerrado sensu stricto no Brasil Central - DF ${ }^{1}$
}

\author{
EDSON S. LIMA ${ }^{2,5}$, JEANINE M. FELFILI'², BEATRIZ S. MARIMON ${ }^{3}$ e ALDICIR SCARIOT ${ }^{4}$
}

(recebido: 21 de fevereiro de 2002; aceito: 25 de junho de 2003)

\begin{abstract}
Diversity, structure and spatial distribution of palms in a cerrado sensu stricto in Central Brazil - DF). The diversity, structure and spatial distribution of palms in a cerrado sensu stricto was studied. Data were collected in 21 plots of $20 \times 50 \mathrm{~m}$ distributed randomly over a $152 \mathrm{ha}$ area $(400 \times 3800 \mathrm{~m})$. The diameters were taken at $0.10 \mathrm{~m}$ above the level of the soil. The reproductive stage and height per individual were recorded. Six species of palms was identified and a total 1671 individuals in 2.1 ha. The total density of the species was 796 palms.ha $^{-1}$, in particular Syagrus flexuosa (Mart.) Becc. (41.2\%), S. petraea (Mart.) Becc. (21.7\%) and S. comosa (Mart.) Mart. (11.2\%). The most frequent were S. flexuosa and Allagoptera leucocalyx (Drude) O. Kuntze. The total basal area was $0.51 \mathrm{~m}^{2} . \mathrm{ha}^{-1}$. S. flexuosa showed the greatest VI. The height and diameter distributions were almost unimodal, with all species. The coefficient of correlation between diameter and height was low $(r=0.0002$ to 0.1247$)$. Syagrus comosa produced the largest height value $(380 \mathrm{~cm})$. Butia archeri stood out in diameter $(6.8-21 \mathrm{~cm})$. All species were fertile, particularly $B$. archeri $(100 \%)$. The pattern of dispersal of all species was aggregated. A classification by TWINSPAN (eigenvalue 0.501) separated B. archeri, S. comosa, and S. petraea as preferential for a group of plots and A. campestris, A. leucocalyx, and S. flexuosa as indifferent. It was concluded that the cerrado contains a rich and abundant flora of palms with morphological and structural differences that requires further research to determine patterns of diversity and structure of palms for the biome.
\end{abstract}

Key words - Brazil, cerrado, diversity, palms, structure

RESUMO - (Diversidade, estrutura e distribuição espacial de palmeiras em um cerrado sensu stricto no Brasil Central - DF). A diversidade, estrutura e distribuição espacial de palmeiras em um cerrado sensu stricto foi estudada. Os dados foram coletados em 21 parcelas de $20 \times 50 \mathrm{~m}$ distribuídas aleatoriamente em 152 ha $(400 \times 3800 \mathrm{~m})$. Os diâmetros foram adquiridos a $0,10 \mathrm{~m}$ acima do nível do solo. Registraram-se o estágio reprodutivo e a altura dos indivíduos. Foram identificadas seis espécies de palmeiras, totalizando 1671 indivíduos em 2,1 ha. A densidade total das espécies foi de 795,7 palmeiras.ha ${ }^{-1}$, destacando-se Syagrus flexuosa (Mart.) Becc. (41,2\%), S. petraea (Mart.) Becc. (21,7\%) e S. comosa (Mart.) Mart. (11,2\%). As mais freqüentes foram S. flexuosa e Allagoptera leucocalyx (Drude) O. Kuntze. A área basal total foi de $0,51 \mathrm{~m}^{2}$.ha' ${ }^{-1}$. S. flexuosa teve o maior VI. A distribuição de alturas e diâmetros foi quase unimodal para todas as espécies. O coeficiente de correlação entre diâmetro e altura foi baixo $(r=0,0002$ a 0,1247$)$. Syagrus comosa apresentou a maior altura $(380 \mathrm{~cm})$. Butia archeri destacou-se em diâmetro (6,8-21 cm). Todas as espécies apresentaram-se férteis, destacando-se B. archeri (100\%). Todas as espécies tiveram padrão de dispersão agregado. Uma classificação por TWINSPAN (autovalor 0,501), separou dois grupos: B. archeri, $S$. comosa e S. petraea, como preferenciais para um grupo de parcelas, e A. campestris, A. leucocalyx e S. flexuosa, como menos agregada. Conclui-se que o cerrado apresenta uma rica e abundante flora de palmeiras com diferenças morfológicas e estruturais que precisam ser melhor conhecidas para se determinarem padrões da diversidade e estrutura de palmeiras para o bioma.

Palavras-chave - Brasil, cerrado, diversidade, estrutura, palmeiras

\section{Introdução}

As palmeiras apresentam ampla distribuição, ocorrendo principalmente nos trópicos e subtrópicos. Exibem hábitos variados, desde estipes subterrâneos e

1 Parte da dissertação de mestrado de E.S. Lima.

2. Universidade de Brasília, Departamento de Engenharia Florestal, Caixa Postal 04357, 70 919-970 Brasília, DF, Brasil.

3. Universidade do Estado de Mato Grosso, Departamento de Ciências Biológicas, 78 690-000 Nova Xavantina, MT, Brasil.

4. Cenargen / Embrapa, Caixa Postal 02372, 70770-900 Brasília, DF, Brasil.

5._Autor para correspondência: eslima@unb.br de pequeno porte até formas arborescentes e raramente trepadeiras, com ou sem espinhos no caule e folhas (Hendersan 1995, Martins 2000). A família Arecaceae tem aproximadamente 189 gêneros e 3000 espécies (Uhl \& Dransfield 1999), sendo que Lorenzi et al. (1996) citam 29 gêneros e 132 espécies para o Brasil.

Embora as palmeiras sejam componentes importantes na vegetação do cerrado, poucos são os estudos relacionados à estrutura das populações. Os trabalhos florísticos e fitossociológicos da camada lenhosa do cerrado, em sua maioria, descartam palmeiras do estudo e isso se deve muitas vezes à metodologia aplicada, considerando que muitas delas 
são de estipes subterrâneos. Estes estudos têm priorizado as espécies que apresentam hábito arborescente (Ribeiro et al. 1985, Felfili \& Silva Júnior 1992, Oliveira Filho et al. 1989, Felfili et al. 1994, Salis et al. 1994, Carvalho et al. 1995, Felfili et al. 1997, Marimon et al. 1998, Silva \& Nogueira 1999, Marimon et al. 2001). Estudos sobre palmeiras têm sido conduzidos, principalmente, com relação à biologia reprodutiva (Scariot et al. 1991, 1995, Rosa et al. 1998) e mortalidade de plântulas, predação e dispersão de sementes (Scariot 1998a, 2000). Entretanto, alguns pesquisadores listaram o maior número possível de espécies para várias regiões do país (Heringer et al. 1977, Goodland 1979, Medeiros Costa \& Panizza 1983, Savage \& Ashton 1983, Filgueiras \& Pereira 1994, Medeiros Costa 1994, Mendonça et al. 1998, Martins 2000).

As palmeiras destacam-se do ponto de vista natural, econômico e ecológico. Além de entrarem rotineiramente na alimentação do homem na forma de frutos e palmito e até de produtos elaborados, como doces, bebidas e óleos, artesanatos de espécies dos gêneros Orbygnia, Syagrus, Acrocomia e Mauritia, são freqüentemente comercializados em feiras e mercados de muitas cidades do Brasil Central (Pereira 1996). Os Xavantes utilizam as palhas em artesanatos e construção de suas casas, a polpa e sementes como alimento e ingredientes para pinturas, e os estipes em suas tradicionais corridas de tora. Várias espécies da fauna silvestre alimentam-se de suas folhas, polpa dos frutos (canídeos) e sementes (roedores de pequeno e médio porte, psitacídeos, etc.).

Estudos sobre diversidade e estrutura da vegetação nas comunidades de cerrado têm sido feitos (Filgueiras et al. 1998, Pires et al. 1999, Felfili et al. 2001, Felfili \& Felfili 2001), e uma boa idéia sobre padrões estruturais das populações tem emergido. Porém, o conhecimento desses padrões para as palmeiras do cerrado é escasso ou inexistente. Este trabalho teve como objetivo descrever e analisar a diversidade, estrutura das populações e distribuição espacial de espécies de palmeiras em uma área de cerrado sensu stricto na Fazenda Água Limpa (FAL), Distrito Federal.

\section{Material e métodos}

Área de estudo - O estudo foi conduzido em uma área de cerrado sensu stricto, na Fazenda Água Limpa - FAL (15 56 '$15^{\circ} 59^{\prime} \mathrm{S}$ e $\left.47^{\circ} 55^{\prime}-47^{\circ} 58^{\prime} \mathrm{W}\right)$. A FAL tem altitude média de $1.100 \mathrm{~m}$ e clima do tipo Aw, segundo a classificação de Köppen (Nimer 1989), com uma precipitação média anual de $1.600 \mathrm{~mm}$, e o pico da estação seca entre julho a setembro. O solo na área de estudo é do tipo Latossolo VermelhoAmarelo, pobre em nutrientes e com elevado teor de alumínio (Furley 1985, Haridasan 1990). A vegetação é composta por um estrato graminoso contínuo com cerca de $50 \mathrm{~cm}$ e outro lenhoso descontínuo com árvores de 3-5 $\mathrm{m}$ e algumas emergentes que podem atingir até $12 \mathrm{~m}$ de altura. Mais detalhes sobre as características da área pode ser encontrados em Felfili et al. (2000).

Amostragem - Os dados foram coletados em 21 parcelas permanentes de $20 \times 50 \mathrm{~m}\left(1.000 \mathrm{~m}^{2}\right.$ cada $)$ distribuídas aleatoriamente em uma faixa de $400 \times 3.800 \mathrm{~m}$ de cerrado sensu stricto. Para efeito de demarcação, foi feita uma picada central onde constam indicações sobre o posicionamento das parcelas no campo, onde a vegetação lenhosa vem sendo estudada desde 1985 (Felfili \& Silva Júnior 1992, Felfili et al. 2000).

Foram identificados e medidos todos os indivíduos adultos e jovens de palmeiras. Consideraram-se como adultos, para as espécies arborescentes, os que apresentavam estipe exposto e, para as espécies de estipe subterrâneo, todos foram considerados adultos. Para as espécies com estipe aéreo, o diâmetro foi determinado em centímetros a $0,10 \mathrm{~m}$ acima do nível do solo com o auxílio de uma suta metálica, exceto para aqueles indivíduos cujo estipe ainda se encontrava abaixo da superfície do solo. Os diâmetros das espécies de estipe subterrâneo não foram mensurados. A altura foi medida da base até o ponto mais alto das folhas de todas as palmeiras. Os indivíduos foram classificados como solitário ou em touceira, dependendo do hábito. Os indivíduos de cada touceira foram medidos separadamente em função do perfilhamento (reprodução vegetativa) ocorrer abaixo da superfície do solo. Foram considerados como indivíduos férteis aqueles que apresentaram qualquer tipo de estrutura reprodutiva, desde que estivesse viva (bráctea peduncular, inflorescência e infrutescência), registrando-se o estágio reprodutivo.

Análise dos dados - Para estimar o padrão de distribuição espacial das palmeiras foi calculado o Índice de Dispersão de Morisita $\left(I_{d}\right)$ (Brower \& Zar 1984), para cada espécie. A significância do valor do $I_{d}$ foi testada a partir da estatística $F$, com nível de confiança de 0,05 (Poole 1974).

Uma classificação por TWINSPAN ("Two-way indicator species analysis") foi feita, onde o método faz uma classificação hierárquica divisiva das espécies em função das parcelas em que estas ocorrem e das parcelas em função das espécies que nelas ocorrem, para identificar espécies preferenciais (aquelas que preferem alguns locais com determinadas características ambientais) ou indiferentes (Hill 1979). A técnica de classificação organiza dados da comunidade baseada exclusivamente na abundância de espécies, independente dos dados ambientais, sendo a interpretação ambiental uma etapa independente (Felfili 1998).

Os parâmetros fitossociológicos considerados foram os comumente utilizados em levantamentos florestais 
quantitativos (Curtis \& McIntosh 1950, 1951). As distribuições de altura e diâmetro foram avaliadas para cada espécie, sendo que os intervalos de classe foram calculados de acordo com Spiegel (1976).

\section{Resultados}

Suficiência da amostragem - Na primeira parcela já ocorreram todas as espécies de palmeiras identificadas na área de estudo, indicando a abrangência da amostragem para o parâmetro número de espécies. $\mathrm{O}$ erro padrão da amostragem, para densidade, foi calculado para uma população infinita, sendo encontrada uma média de 795 ind.ha $^{-1}( \pm 13,5)$ com intervalo de confiança (IC) a $95 \%=\left[518\right.$ ind.ha $^{-1} \leq \bar{x} \leq$ 1.082 ind.ha $^{-1}$.

Florística e fitossociologia - Foram encontradas seis espécies de palmeiras (Allagoptera campestris (Drude) O. Kuntze, A. leucocalyx (Drude) O. Kuntze, Butia archeri (Glassman) Glassman, Syagrus comosa (Mart.) Mart., S. flexuosa (Mart.) Becc. e S. petraea (Mart.) Becc.), totalizando 1.671 indivíduos em 2,1 ha amostrados (tabela 1), representando 796 palmeiras.ha ${ }^{-1}$. As mais abundantes foram $S$. flexuosa $(41,2 \%$ do total de indivíduos amostrados), S. petraea $(21,7 \%)$ e $S$. comosa $(11,2 \%)$. As mais freqüentes foram S. flexuosa e A. leucocalyx ( $20 \%$ das parcelas cada), A. campestris (17\%) e $S$. comosa $(16 \%)$. A área basal total das três espécies com estipes aéreos foi de $0,51 \mathrm{~m}^{2} \cdot \mathrm{ha}^{-1}$. O cálculo do índice de valor de importância somente foi possível para as espécies com estipe aéreo, pois este parâmetro depende da medida do diâmetro, destacando-se, S. flexuosa e B. archeri (tabela 1).

Distribuição de classes - A distribuição de alturas foi quase unimodal para todas as espécies (figura 1). A maioria das espécies teve maior número de indivíduos nas classes de altura intermediárias, que ocorreu principalmente entre $31-40 \mathrm{~cm}$, para Allagoptera campestris, e $81-90 \mathrm{~cm}$, para A. leucocalyx, enquanto que para as demais espécies ocorreram em classes de alturas intermediárias a estes (figura 1).

A maioria dos indivíduos de Syagrus comosa, S. flexuosa e Butia archeri ocorreu nas classes intermediárias de diâmetros (figura 2). Syagrus comosa foi a única espécie que apresentou o maior número de indivíduos na primeira classe. As palmeiras não apresentam desenvolvimento secundário típico ou cambial, portanto, as maiores densidades foram representadas nas classes intermediárias para cada espécie, onde $S$. flexuosa enquadrou-se claramente nesta situação (figura 2).

A corrrelação $(r)$ entre diâmetro e altura foi baixa, variando de 0,0002 em Syagrus comosa a 0,1247 em Butia archeri.

Syagrus comosa foi a mais alta das seis espécies, com indivíduos adultos medindo entre $89-380 \mathrm{~cm}$ $(\mathrm{Md}=62)$ e diâmetro entre 6,5-17,5 cm (tabela 2, figura $3 \mathrm{~A} \mathrm{e} \mathrm{B).} \mathrm{Allagoptera} \mathrm{campestris} \mathrm{foi} \mathrm{a} \mathrm{menor,} \mathrm{com} \mathrm{altura}$ entre 20-94 cm $(\mathrm{Md}=40)$ (tabela 2, figura 3A). Butia archeri teve os maiores diâmetros variando entre

Tabela 1. Parâmetros fitossociológicos das espécies de palmeiras encontradas no cerrado sensu stricto, na Fazenda Água Limpa, Brasília, DF. DA = densidade absoluta; DR = Densidade relativa (\%); FA = frequência absoluta; FR = freqüência relativa $(\%)$; DoA = dominância absoluta; DoR = dominância relativa (\%); e IVI = índice de valor de importância. Os parâmetros relativos para obtenção do IVI abrangeram apenas as populações de estipe aéreos (- = espécies com estipes subterrâneos).

Table 1. Phytosociological parameters of palm species found in a cerrado sensu stricto at Água Limpa farm, Brasília, DF. DA = absolute density; $\mathrm{DR}=$ relative density; $(\%) ; \mathrm{FA}=$ absolute frequency; $\mathrm{FR}=$ relative frequency $(\%)$; DoA = absolute dominance; DoR $=$ relative dominance $(\%)$; and IVI = importance value index. Relative paramenters for obtaining IVI includes only populations with arborescent palms (- = species with underground stems).

\begin{tabular}{lccccccc}
\hline Espécie & DA & DR & FA & FR & DoA & DoR & IVI \\
\hline Allagoptera campestris & 59,05 & 7,42 & 80,95 & 17 & - & - & - \\
Allagoptera leucocalyx & 85,71 & 10,77 & 95,24 & 20 & - & - & - \\
Butia archeri & 61,43 & 7,72 & 66,67 & 14 & 0,16 & 31,19 & 72,04 \\
Syagrus comosa & 89,05 & 11,18 & 76,19 & 16 & 0,05 & 10,03 & 60,65 \\
Syagrus flexuosa & 327,60 & 41,21 & 95,24 & 20 & 0,30 & 58,78 & 167,31 \\
Syagrus petraea & 172,86 & 21,71 & 61,91 & 13 & - & - & - \\
Total & 795,70 & 100,00 & 476,20 & 100 & 0,51 & 100,00 & 300,00 \\
\hline
\end{tabular}



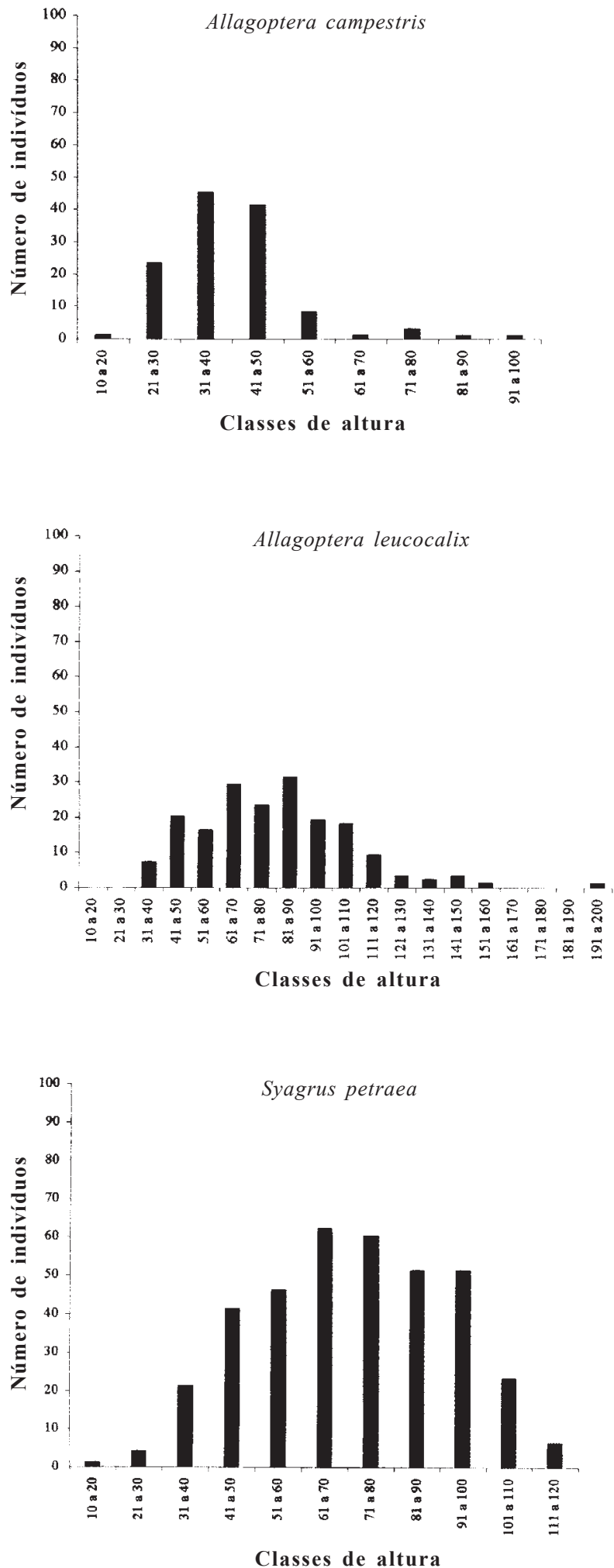

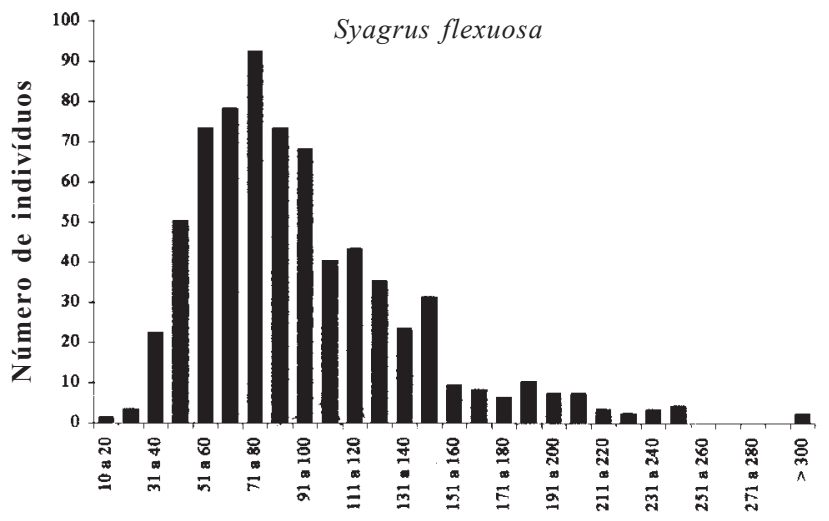

Classes de altura

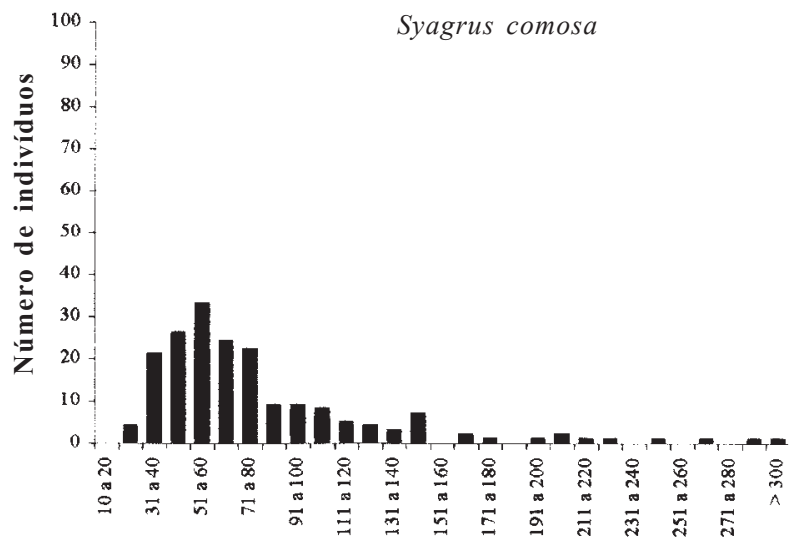

Classes de altura

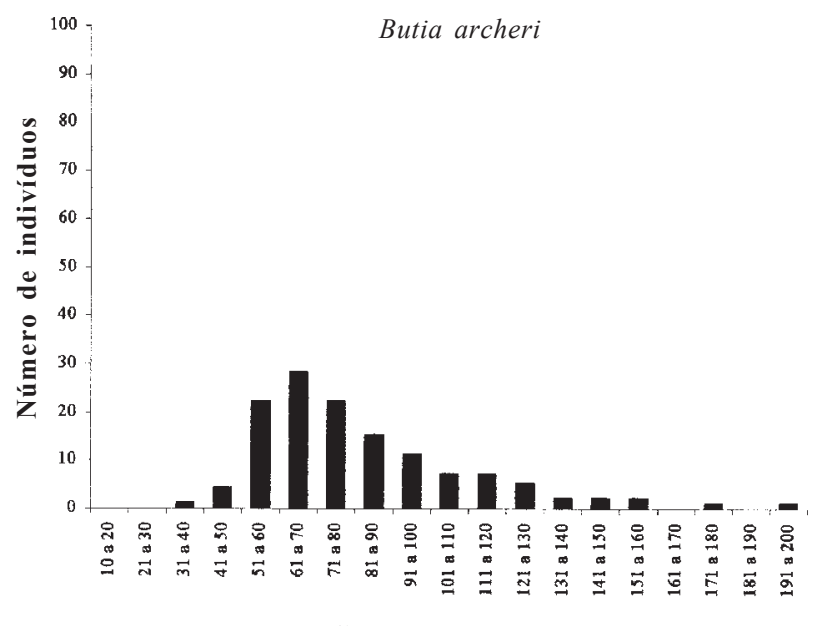

Classes de altura

Figura 1. Distribuição de altura (cm) de seis espécies de palmeiras no cerrado sensu stricto na Fazenda Água Limpa, Brasília, DF.

Figure 1. Distribution and height (cm) of six palm species in a cerrado sensu stricto at “Água Limpa” Farm, Brasília, DF. 

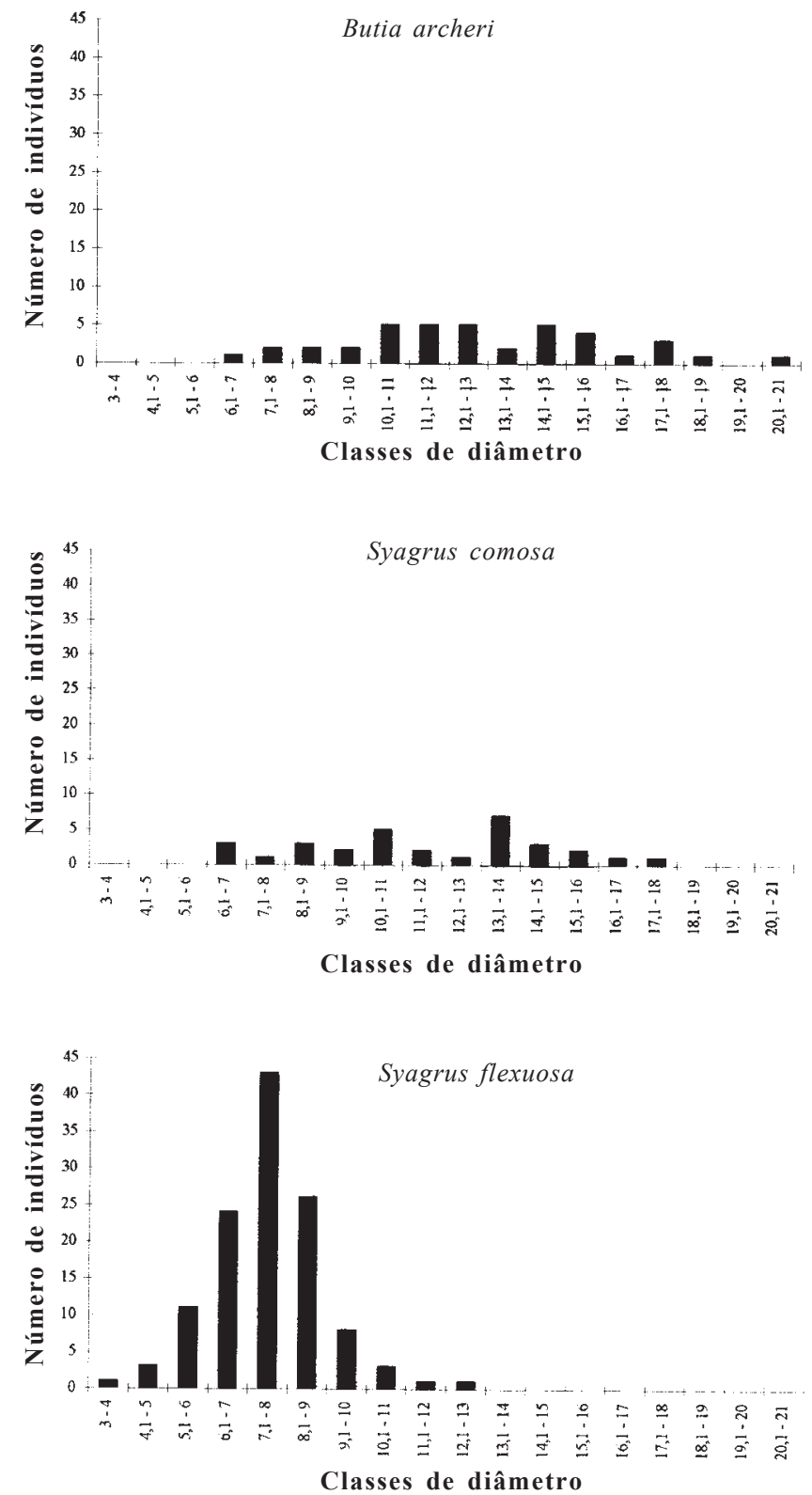

Figura 2. Distribuição de diâmetros de três espécies de palmeiras no cerrado sensu stricto na Fazenda Água Limpa, Brasília, DF. Observe a escala das figuras.

Figure 2. Distribution of diameters of three palm species in a cerrado sensu stricto at "Água Limpa" Farm, Brasília, DF. See figure scales.
6,8-21 cm, seguido por Syagrus comosa com $6,5-17,5 \mathrm{~cm}$ (tabela 2, figura 3B).

Razão jovem-adulto - $\mathrm{O}$ maior número de indivíduos jovens foi verificado para Syagrus flexuosa (tabela 3). Foram registrados para esta espécie 688 indivíduos, dos quais 634 (92,2\%) encontravam-se organizados em 86 touceiras e $54(7,8 \%)$ solitários. Verificou-se uma média de 7,4 $( \pm 4,6)$ indivíduos por touceira e considerando somente adultos $1,3( \pm 1,7)$. Todas as espécies de palmeiras apresentaram indivíduos férteis, destacandose Butia archeri, onde $100 \%$ dos indivíduos adultos encontravam-se em estado reprodutivo e $S$. petraea $(36,1 \%)$. As demais espécies apresentaram baixos percentuais de indivíduos férteis (tabela 3). Syagrus comosa apresentou-se fértil a uma altura mínima de $45 \mathrm{~cm}$, B. archeri $55 \mathrm{~cm}$ e $S$. flexuosa $76 \mathrm{~cm}$.

Padrão de dispersão - Todas as espécies de palmeiras tiveram o valor do Índice de Dispersão de Morisita significativamente maior do que um, demonstrando padrão de dispersão agregado (tabela 4), embora tenham ampla distribuição na área de estudo.

A primeira divisão obtida pelo método TWINSPAN (autovalor 0,501), separou dois grupos principais, o primeiro Butia archeri, Syagrus comosa e S. petraea caracterizou-se como espécies preferenciais para um grupo de parcelas e o segundo Allagoptera campestris, A. leucocalyx e S. flexuosa como espécies indiferentes. $\mathrm{O}$ primeiro grupo de palmeiras caracterizou-se pelas espécies menos freqüentes e o segundo pelas mais freqüentes na área de estudo (tabela 1).

\section{Discussão}

A riqueza e a densidade ( 6 espécies e 796 ind.ha ${ }^{-1}$ ) de palmeiras na área estudada foi alta quando comparadas com as espécies lenhosas representantes das famílias Vochysiaceae (5 e 194) e Leguminosae (6 e $145)$, consideradas as de maior riqueza e densidade por Felfili et al. (2000) na mesma área de estudo. Geralmente, em estudos fitossociológicos da vegetação lenhosa do cerrado encontram-se poucas espécies abundantes e muitas espécies raras. Com palmeiras do cerrado, a diferença verificada entre as densidades não foi tão acentuada, pois a espécie mais abundante representou $41 \%$ da densidade total $(327,6$ ind./ha) e a menos abundante 7\% (59 ind.ha $\left.{ }^{-1}\right)$. Nesta mesma área, as 10 espécies lenhosas mais abundantes representaram $53 \%$ da densidade total (Felfili et al. 2000). Oliveira 

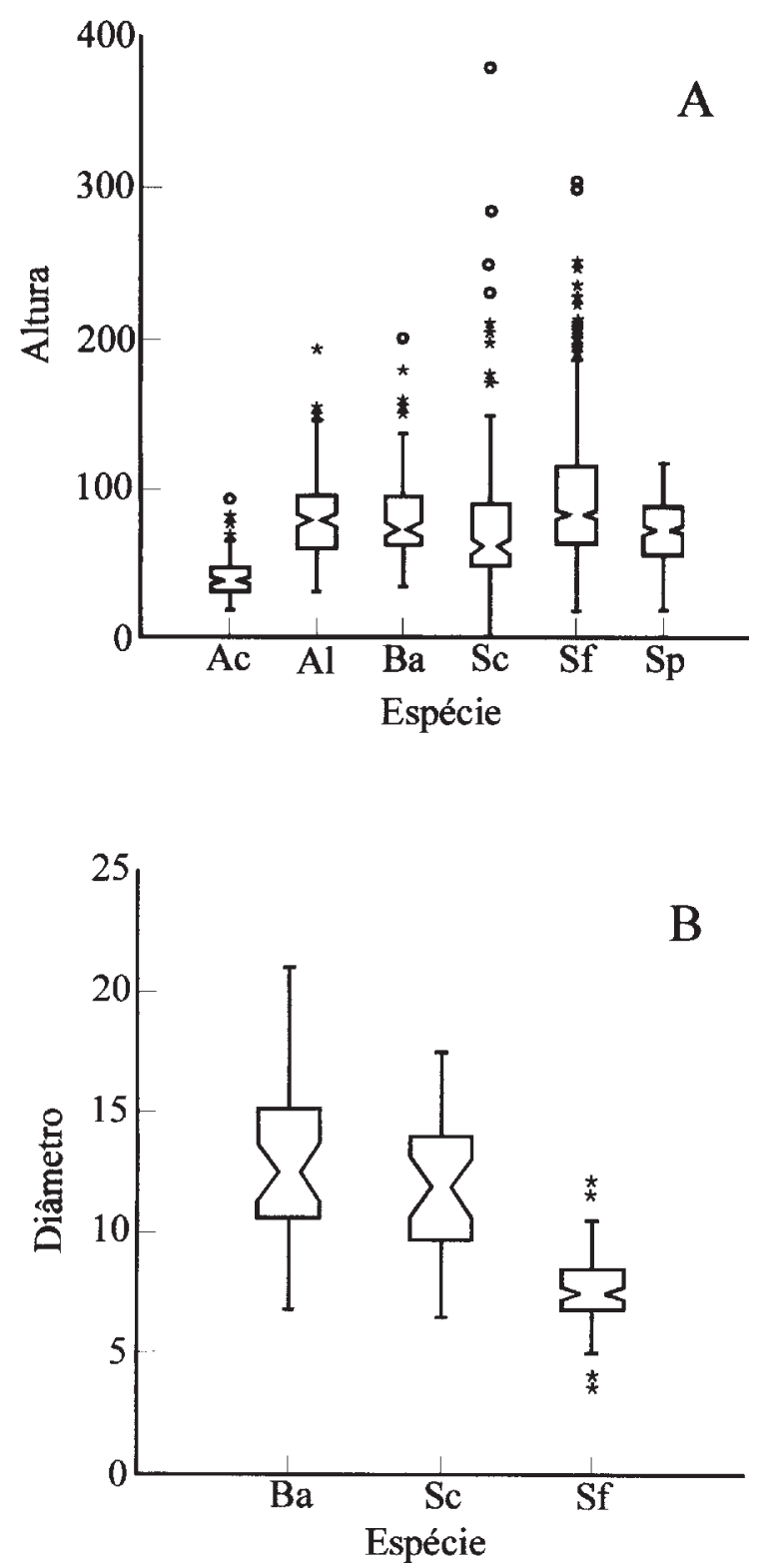

Figura 3. Diagramas box-plots das características morfológicas de seis espécies de palmeiras no cerrado sensu stricto da Fazenda Água Limpa, Brasília, DF. A = altura (cm); e $\mathrm{B}=$ diâmetro $(\mathrm{cm})$. Ac $=$ Allagoptera campestris; $\mathrm{Al}=$ A. leucocalyx $; \mathrm{Ba}=$ Butia archeri; $\mathrm{Sc}=$ Syagrus comosa $; \mathrm{Sf}$ $=S$. flexuosa; e $\mathrm{Sp}=S$. petraea . No gráfico box-plot, os box comprimidos compreendem 50\% dos dados; a marca central no box indica a mediana; asteriscos são "valores distantes" e círculos são "valores muito distantes".

Figure 3. Box-plots of morphological characteristics of six palm species in a cerrado sensu stricto at Água Limpa farm, Brasília, DF. A = height $(\mathrm{cm})$; and $\mathrm{B}=\operatorname{diameter}(\mathrm{cm})$. Ac = Allagoptera campestris; $\mathrm{Al}=$ A. leucocalyx $; \mathrm{Ba}=$ Butia archeri; $\mathrm{Sc}=$ Syagrus comosa; $\mathrm{Sf}=$ S. flexuosa; and $\mathrm{Sp}=$ S. petraea. In the box-plot graph, the long box comprises $50 \%$ of the data; the central line marks the median; asterics are "outside values" and circles are "far outside values".
Tabela 2. Medianas e amplitude das variáveis alométricas das seis espécies de palmeiras amostradas no cerrado sensu stricto na Fazenda Água Limpa, Brasília, DF.

Table 2. Medians and amplitude of alometric variables of six palm species sampled in a cerrado sensu stricto at "Água Limpa"Farm, Brasília, DF.

\begin{tabular}{lcc}
\hline Espécie & $\begin{array}{c}\text { Mediana } \\
\text { da altura }(\mathrm{cm})\end{array}$ & $\begin{array}{c}\text { Amplitude } \\
\text { do diâmetro }(\mathrm{cm})\end{array}$ \\
\hline Allagoptera campestris & 40 & - \\
Allagoptera leucocalyx & 80 & - \\
Butia archeri & 74 & $6,8-21,0$ \\
Syagrus comosa & 62 & $6,5-17,5$ \\
Syagrus flexuosa & 84 & $5,0-12,2$ \\
Syagrus petraea & 73 & - \\
\hline
\end{tabular}

Filho et al. (1989), estudando a variação florística em uma área de cerrado no estado de Mato Grosso, registraram diferentes densidades para Syagrus comosa em quatro subtipos de cerrado (700 ind.ha ${ }^{-1}$ em um cerrado rochoso a 53 ind.ha $^{-1}$ em um cerrado alto de interflúvio), onde esteve quase sempre nas primeiras posições na hierarquia fitossociológica. $\mathrm{O}$ posicionamento das espécies na hierarquia de densidades varia entre diferentes locais, mesmo para áreas vizinhas. Como por exemplo, em um cerrado do Jardim Botânico de Brasília, vizinho a FAL, a densidade de $S$. comosa foi de 177 ind.ha $^{-1}$ (Dusi 1989), muito acima do verificado neste trabalho. A espécie mais abundante de palmeira em uma floresta de terra firme na Amazônia representou $64 \%$ da densidade total $\left(80,7\right.$ ind.ha $\left.^{-1}\right)$ em terras baixas e $66 \%\left(36,28\right.$ ind.ha $\left.^{-1}\right)$ em terras altas (Peres 1994). A densidade média de palmeiras verificada em fragmentos de floresta contínua na Amazônia Central foi de 7.188 ind.ha $^{-1}$ (Scariot 1998b) e 3.975 ind.ha $^{-1}$ em uma floresta sazonalmente inundada no leste da Amazônia (Scariot et al. 1989). Em diferentes locais na Floresta Amazônica a densidade de palmeiras também é variável.

Syagrus flexuosa foi a espécie de maior valor de importância, em função do elevado número de indivíduos presentes na área de estudo e Butia archeri, devido ao maior diâmetro individual. Em vários estudos fitossociológicos da vegetação lenhosa do cerrado, algumas espécies de palmeiras têm ocupado posições relevantes em valor de importância (VI) (Ribeiro et al. 1985, Oliveira Filho et al. 1989). Marimon et al. (1998) em um estudo conduzido na região de Nova Xavantina, verificaram que $S$. flexuosa ocupou a décima posição 
Tabela 3. Número de indivíduos jovens e adultos, férteis e vegetativos em seis espécies de palmeiras no cerrado sensu stricto na Fazenda Água Limpa, Brasília, DF.

Table 3. Number of adult and juvenile individuals, fertile and vegetative in six palm species in a cerrado sensu stricto at “Água Limpa” Farm, Brasília, DF.

\begin{tabular}{lcrrc}
\hline Espécie & Jovem & $\begin{array}{r}\text { Adulto } \\
\text { Fértil } \\
(\%)\end{array}$ & $\begin{array}{c}\text { Vegetativo } \\
(\%)\end{array}$ \\
\hline Allagoptera campestris & - & 124 & 18,5 & 81,5 \\
Allagoptera leucocalyx & - & 180 & 5,5 & 94,5 \\
Butia archeri & 90 & 39 & 30,2 & 69,8 \\
Syagrus comosa & 156 & 31 & 5,9 & 94,1 \\
Syagrus flexuosa & 571 & 117 & 5,1 & 94,9 \\
Syagrus petraea & - & 363 & 36,1 & 63,9 \\
\hline
\end{tabular}

em VI e S. comosa, identificada como Butia leiospatha (Mart.) Bacc., a décima quarta, entre a vegetação lenhosa do cerrado. Butia archeri ocupou a segunda posição em VI, porém não tem se destacado em Silva \& Nogueira (1999). No presente estudo, Syagrus comosa foi a espécie de menor VI, embora tenha sido bem representada no estudo de Ribeiro et al. (1985). Os trabalhos publicados evidenciam variações consideráveis nos VI das espécies estudadas.

Butia archeri, Syagrus petraea e $S$. comosa apresentaram elevadas densidades e menores freqüências quando comparadas às outras espécies estudadas. Esta situação sugere que palmeiras do cerrado, mesmo tendo padrão agregado, apresentam diferenças na distribuição espacial. Marimon et al. (1998) verificaram condição similar para S. flexuosa e S. comosa em Nova Xavantina, MT.

A distribuição de altura das palmeiras caracterizou-se pelo acúmulo de indivíduos nas classes intermediárias, o que pode sugerir que o recrutamento seja reduzido. Marimon et al. (2001) constataram situação similar para Mauritia flexuosa L. em uma mata de galeria, onde sugeriram que o ciclo de vida da espécie não esteja se completando, pois a regeneração natural não está conseguindo estabelecer e futuramente as classes posteriores poderão estar pouco representadas. Dusi (1989) verificou situação similar para Syagrus comosa e $S$. flexuosa, embora o maior número de estipes tenha sido apresentado para a segunda classe de altura (0,1 a 0,2 m). Entretanto, é importante lembrar que o autor não inventariou indivíduos jovens. Estudos sobre recrutamento destas espécies, provavelmente auxiliariam o entendimento deste padrão de distribuição de alturas. Apesar das palmeiras ocorrerem em formações com dossel aberto de cerrado, a etapa de germinação e desenvolvimento inicial pode ocorrer sobre sombreamento ocasionado pela vegetação graminosa e herbácea e pela cobertura de copas em alguns locais do cerrado sensu stricto. Clark \& Clark (1987) sugerem que este padrão de distribuição ocorra através de recrutamento episódico e Felfili (1995) sugere que padrões como estes ocorram com espécies que necessitam de grande quantidade de luz (clareiras) para se estabelecer.

A distribuição de diâmetros das três espécies com estipe aéreo demonstrou uma maior concentração de indivíduos nas classes intermediárias, provavelmente em virtude de palmeiras não apresentarem crescimento secundário típico ou cambial, podendo diminuir o diâmetro através da morte e destruição dos tecidos externos do estipe. Esta diminuição, às vezes sutil, deve ocorrer devido fogos periódicos, comuns no cerrado. Este fato pôde ser reforçado pela baixa relação hipsométrica $(r=0,0002-0,1247)$ com a altura.

Todas as espécies apresentaram um padrão de dispersão agregado. É esperado que espécies que apresentam altas freqüências se distribuam de forma aleatória. No entanto, espécies como Allagoptera campestris, A. leucocalyx e Syagrus flexuosa, apesar das elevadas freqüências, distribuíram-se de forma agrupada. Uma classificação por TWINSPAN, separou as espécies menos freqüentes daquelas mais freqüentes. Estas, provavelmente, corresponderam a um padrão de agrupamento mais definido, ocorrendo em touceiras

Tabela 4. Valores do Índice de Dispersão de Morisita $\left(I_{d}\right)$ e da estatística $F$, e padrão de distribuição das espécies de palmeiras encontradas no cerrado sensu stricto, na Fazenda Água Limpa, Brasília, DF. $I_{d}>1=$ distribuição agregada. $\mathrm{F}_{20, \propto}=1,57$.

Table 4. Values of Morisita Dispersion Index $\left(I_{d}\right)$ and of $F$ statistics, and distribution patterns of palm species found in the cerrado sensu stricto at “Água Limpa” Farm, Brasília, DF. $I_{d}>1$ = aggregated distribution. $\mathrm{F}_{20, \propto}=1,57$.

\begin{tabular}{lccc}
\hline Espécie & $I_{d}$ & $F$ & $\begin{array}{c}\text { Distribuição } \\
\text { espacial }\end{array}$ \\
\hline Allagoptera campestris & 1,13 & 1,81 & agrupada \\
Allagoptera leucocalyx & 1,18 & 2,57 & agrupada \\
Butia archeri & 1,13 & 1,86 & agrupada \\
Syagrus comosa & 1,08 & 1,75 & agrupada \\
Syagrus flexuosa & 1,16 & 6,36 & agrupada \\
Syagrus petraea & 1,32 & 6,75 & agrupada \\
\hline
\end{tabular}


mais distanciadas. Este fato, embora não caracterizado, pode estar também relacionado com alguma variável ambiental ou com taxas de predação, capacidade de dispersão, germinação das sementes e estabelecimento das plântulas. Syagrus flexuosa, por exemplo, possui polpa apreciada por várias espécies da fauna, as quais contribuem para a dispersão de suas sementes evitando muitas vezes que sejam predadas nas proximidades da planta mãe. É comum a presença de frutos de palmeiras nas fezes de várias espécies da fauna do cerrado como lobinho (Cerdocyon thous), ema (Rhea americana), porco-do-mato (Tayassu spp.) e anta (Tapirus terrestris). Dalponte (1997) verificou que endocarpos de palmeiras contribuiram com $15,4 \%$ dos itens vegetais consumidos pela raposa-do-campo (Lycalopex vetulus) e Dalponte \& Lima (1999) verificaram que endocarpos de Astrocaryum campestre Mart., Allagoptera campestris e Syagrus comosa estavam presentes nas fezes desse canídeo, sendo que endocarpos de Astrocaryum sp. são dispersos nas fezes do lobo-guará (Chrysocyon brachyurus) (Azevedo \& Gastal 1997). Animais que constituem latrinas, poderiam estar contribuindo com a germinação de um grande número de sementes em locais relativamente próximos. Entretanto, estudos sobre a viabilidade das sementes de palmeiras que passaram pelo trato digestivo de animais deverão ser feitos para avaliar sua viabilidade e a legitimidade do dispersor.

O maior número de indivíduos jovens foi registrado para Syagrus flexuosa e esse fato provavelmente, deve-se à capacidade de propagação vegetativa da espécie que ocorre na natureza em touceiras, onde estas estão interligadas abaixo da superfície do solo. Portanto, cada touceira poderia ser considerada como um único indivíduo (genet). Se assim fosse considerado, a espécie teria apenas 140 genets, alterando substancialmente a densidade apresentada nos resultados.

A estrutura populacional das seis espécies de palmeiras estudadas foi distinta e a densidade populacional alta, quando comparada com a vegetação lenhosa do cerrado na mesma área de estudo e na Amazônia em floresta de terra firme. A distribuição espacial das palmeiras foi agregada, apesar de ocorrência ampla na área de estudo. Entretanto, recomenda-se que estudos com palmeiras em várias regiões ao longo do Cerrado sejam desenvolvidos, para que se possa determinar padrões de diversidade, estrutura e distribuição espacial das palmeiras no bioma.

Agradecimentos - Os autores agradecem ao Dr. Tarciso S. Filgueiras pela orientação na coleta de dados, Kennia Mara
Ramos, Elisa Maria Meirelles, Renes Monteiro, Julio Dalponte e Newton Rodrigues pelo auxílio na coleta de dados. Ao CNPq, programa PELD, pelo apoio logístico e pela concessão de bolsas.

\section{Referências bibliográficas}

AZEVEDO, F.C.C. \& GASTAL, M.L.A. 1997. Hábito alimentar do lobo guará (Chrysocyon brachyurus), na APA Gama Cabeça de Veado, DF. In Contribuição ao conhecimento ecológico do cerrado (L.L. Leite \& C.H. Saito, org.). Editora da Universidade de Brasília, Brasília, p.238-240.

BROWER, J.E. \& ZAR, J.H. 1984. Field \& laboratory methods for general ecology. Wm. C. Brown Publishers, Iowa.

CARVALHO, D.A., OLIVEIRA FILHO, A.T., VILELA, E.A. \& GAVILANES, M.L. 1995. Estrutura fitossociológica de mata ripária do alto Rio Grande (Bom Sucesso, estado de Minas Gerais). Revista Brasileira de Botânica 22:39-49.

CLARK, D.A. \& CLARK, D.B. 1987. Análisis de la regeneración de árboles del dosel en bosque muy húmedo tropical: aspectos teóricos y práticos. Revista de Biologia Tropical 35:41-54.

CURTIS, J.T. \& MCINTOSH, R.P. 1950. The interrelations of certain analytic and synthetic phytosociological characters. Ecology 31:434-455.

CURTIS, J.T. \& MCINTOSH, R.P. 1951. An upland forest continuum in the prairie-forest border region of Wisconsin. Ecology 32:476-496.

DALPONTE, J.C. 1997. Diet of hoary fox, Lycalopex vetulus, in Mato Grosso, central Brazil. Mammalia 61:537-546.

DALPONTE, J.C. \& LIMA, E.S. 1999. Disponibilidade de frutos e a dieta de Lycalopex vetulus (Carnívora: Canidae) em um cerrado de Mato Grosso, Brasil. Revista Brasileira de Botânica 22:325-332.

DUSI, R.L.M. 1989. Padrão espacial, estrutura de populações e associação de Syagrus comosa Mart. e Syagrus flexuosa Mart. (Palmae) em cerrado no Jardim Botânico de Brasília, DF. Dissertação de mestrado, Universidade de Brasília, Brasília.

FELFILI, J.M. 1995. Growth, recruitment and mortality in the Gama gallery forest in central Brazil over a six-year period (1985-1991). Journal of Tropical Ecology 11:67-83.

FELFILI, J.M. 1998. Determinação de padrões de distribuição de espécies em uma mata de galeria no Brasil Central com a utilização de técnicas de análise multivariada. Boletim do Herbário Ezechias Paulo Heringer 2:35-48.

FELFILI, M.C. \& FELFILI, J.M. 2001. Diversidade alfa e beta no cerrado sensu stricto da Chapada Pratinha, Brasil. Acta Botanica Brasilica 15:243-254.

FELFILI, J.M. \& SILVA JUNIOR, M.C. 1992. Floristic composition, phytosociology and comparison of cerrado and gallery forest at Fazenda Água Limpa, Federal District, Brazil. In Nature and dynamics of forest-savanna boundaries (P.A. Furley, J. Proctor \& J.A. Ratter, eds.). Chapman \& Hall, London, p.393-416. 
FELFILI, J.M., HARIDASAN, M., MENDONÇA, R.C., FILGUEIRAS, T.S., SILVA JUNIOR, M.C. \& REZENDE, A.V. 1994. Projeto biogeografia do bioma cerrado: vegetação \& solos. Cadernos de Geociências 12:75-166.

FELFILI, J.M., SILVA JUNIOR, M.C., REZENDE, A.V., NOGUEIRA, P.E., WALTER, B.M.T., FELFILI, M.C., SILVA, M.A. \& ENCINAS, J.I. 1997. Comparação do cerrado (sensu stricto) nas Chapadas Pratinha e dos Veadeiros. In Contribuição ao conhecimento ecológico do cerrado (L.L. Leite \& C.H. Saito, org.). Editora da Universidade de Brasília, Brasília, p.6-11.

FELFILI, J.M., REZENDE, A.V., SILVA JUNIOR, M.C. \& SILVA, M.A. 2000. Changes in the floristic composition of cerrado sensu stricto in Brazil over a nine-year period. Journal of Tropical Ecology 16:579-590.

FELFILI, J.M., SILVA JUNIOR, M.C., REZENDE, A.V., HARIDASAN, M., FILGUEIRAS, T.S., MENDONÇA, R.C., WALTER, B.M.T. \& NOGUEIRA, P.E. 2001. O projeto biogeografia do bioma Cerrado: hipóteses e padronização da metodologia. In Conservação da biodiversidade em ecossistemas tropicais: avanços conceituais e revisão de novas metodologias de avaliação e monitoramento (I. Garay \& B.F.S. Dias, eds.). Vozes, Petrópolis, p.157-173.

FILGUEIRAS, T.S. \& PEREIRA, B.A.S. 1994. Flora do Distrito Federal. In Cerrado, caracterização, ocupação e perspectivas (M.N. Pinto, ed.). Editora da Universidade de Brasília, Brasília, p.345-404.

FILGUEIRAS, T.S., FELFILI, J.M., SILVA JUNIOR, M.C. \& NOGUEIRA, P.E. 1998. Floristic and structural comparasion of cerrado (sensu stricto) vegetation in Central Brazil. In Forest biodiversity in north, central and south America, and the Caribbean (F. Dallmeier \& J.A. Comiskey, eds.). Unesco/Parthenon, Paris/Carnforth, p.633-648.

FURLEY, P.A. 1985. Notes on the soils and plant communities of Fazenda Água Limpa (Brasília, DF, Brasil). University of Edinburg, Edinburg, Occasional Publications NS 5, $138 \mathrm{p}$.

GOODLAND, R. 1979. Análise ecológica da vegetação do cerrado. In Ecologia do cerrado (R. Goodland \& M.G. Ferri, eds.). Editora da Universidade de São Paulo, São Paulo, p.61-161.

HENDERSON, A. 1995. The palms of the Amazon. Oxford University Press, New York.

HARIDASAN, M. 1990. Solos do Distrito Federal. In Cerrado, caracterização, ocupação e perspectivas (M.N. Pinto, ed.). Editora Universidade de Brasília, Brasília, p.309330.

HERINGER, E.P., BARROSO, G.M., RIZZO, J.A. \& RIZZINI C.T. 1977. Flora do cerrado. In Simpósio sobre o cerrado (M.G. Ferri, coord.). Editora Itatiaia, Brasília, p.211-232.

HILL, M.O. 1979. TWINSPAN - a FORTRAN program for arranging multivariate data in an ordered two way table by classification of the individuals and the attributes. Cornell University, Ithaca.
LORENZI, H., SOUZA, H.M., MEDEIROS-COSTA, J.T., CERQUEIRA, L.S.C. \& VON BEHR, N. 1996. Palmeiras no Brasil Nativas e Exóticas. Editora Plantarum, Nova Odessa.

MARIMON, B.S., VARELLA, R.F. \& MARIMON JÚNIOR, B.H. 1998. Fitossociologia de uma área de cerrado de encosta em Nova Xavantina, Mato Grosso. Boletim do Herbário Ezechias Paulo Heringer 3:82-101.

MARIMON, B.S., FELFILI, J.M., LIMA, E.S. \& RODRIGUES, A.J. 2001. Distribuições de circunferências e alturas em três porções da Mata de Galeria do Córrego Bacaba, Nova Xavantina-MT. Revista Árvore 25:1-9.

MARTINS, R.C. 2000. Arecaceae (Palmae) no Distrito Federal, Brasil. Dissertação de mestrado, Universidade de Brasília, Brasília.

MEDEIROS-COSTA, J.T. \& PANIZZA, S. 1983. Palms of the cerrado vegetation formation of São Paulo state, Brazil. Principes 27:118-125.

MEDEIROS-COSTA, J.T. 1994. A review on the occurrence of Arecaceae (Palmae) in Brazilian cerrado vegetation. Acta Horticulturae 360:73-80.

MENDONÇA, R.C., FELFILI, J.M., WALTER, B.M.T., SILVA JÚNIOR, M.C., REZENDE, A.V. FILGUEIRAS, T.S. \& NOGUEIRA, P.E. 1998. Flora vascular do cerrado. In Cerrado Ambiente e Flora (S.M. Sano \& S.P. Almeida, eds.). Embrapa-CPAC, Planaltina, p.289-539.

NIMER, E. 1989. Climatologia do Brasil. IBGE, Rio de Janeiro.

OLIVEIRA FILHO, A.T., SHEPHERD, G.J., MARTINS, F.R. \& STUBBLEBINE, W.H. 1989. Environmental factors affecting physiognomic and floristic variation in an area of cerrado in central Brazil. Journal of Tropical Ecology 5:413-431.

PEREIRA, B.A.S. 1996. Flora nativa. In Alternativas de desenvolvimento dos cerrados: conservação dos recursos naturais renováveis (B.F.S. Dias, coord.). Fundação Pró-Natureza, Brasília, p.53-57.

PERES, C.A. 1994. Composition, density, and fruiting phenology of arborescent palms in an Amazonian terra firme forest. Biotropica 26:285-294.

PIRES, A., FELFILI, J.M. \& ABREU, A.R. 1999. Florística e fitossociologia do cerrado stricto sensu na APA de Cafuringa-DF. Boletim do Herbário Ezechias Paulo Heringer 4:5-20.

POOLE, R.W. 1974. An introduction to quantitative ecology. McGraw-Hill, New York.

RIBEIRO, J.F., SILVA, J.C.S. \& BATMANIAN, G.J. 1985. Fitossociologia de tipos fisionômicos de cerrado em Planaltina-DF. Revista Brasileira de Botânica 8:131-142.

ROSA, L., CASTELLANI, T.T. \& REIS, A. 1998. Biologia reprodutiva de Butia capitata (Martius) Beccari var. odorata (Palmae) na restinga do município de Laguna, SC. Revista Brasileira de Botânica 21:281-287.

SALIS, S.M., TAMASHIRO, J.Y. \& JOLY, C.A. 1994. Florística e fitossociologia do estrato arbóreo de um remanescente de mata ciliar do rio Jacaré-Pepira, Brotas, SP. Revista Brasileira de Botânica 17:93-103 
SAVAGE, A.J.P. \& ASTON, P.S. 1983. The population structure of the double coconut and some other Seychelles palms. Biotropica 15:15-25.

SCARIOT, A., OLIVEIRA FILHO, A.T. \& LIERAS, E. 1989. Species richness, density and distribution of palms in an eastern Amazonian seazonally flooded forest. Principes 33:172-179.

SCARIOT, A., LIERAS, E. \& HAY, J.D. 1991. Reproductive biology of the palm Acrocomia aculeata in central Brazil. Biotropica 23:12-22.

SCARIOT, A., LIERAS, E. \& HAY, J.D. 1995. Flowering and fruiting phenologies of the Palm Acrocomia aculeata: patterns and consequences. Biotropica 27:168-173.

SCARIOT, A. 1998a. Seed dispersal and predation of the palm Acrocomia aculeata. Principes 42:5-8.
SCARIOT, A. 1998b. Conseqüências da fragmentação da floresta na comunidade de palmeiras na Amazônia Central. Série Técnica IPEF 12:71-86.

SCARIOT, A. 2000. Seedling mortality by litterfall in Amazonian forest fragments. Biotropica 32:662-669

SILVA, M.A. \& NOGUEIRA, P.E. 1999. Avaliação fitossociológica do estrato arbustivo-herbáceo em cerrado stricto sensu após incêndio acidental, no Distrito Federal, Brasil. Boletim do Herbário Ezechias Paulo Heringer 4:65-79.

SPIEGEL, M.P. 1976. Estatística. McGraw-Hill, São Paulo.

UHL, N.W. \& DRANSFIELD, J. 1999. Genera palmarum after ten years. In Evolution, variation, and classification of palms (A. Henderson \& F. Borchsemius, eds.). New York Botanical Garden, New York, p.245-253. 